\title{
Approval voting and Arrow's impossibility theorem
}

\author{
François Maniquet • Philippe Mongin
}

Received: 8 April 2013 / Accepted: 10 September 2014 / Published online: 23 September 2014 (C) Springer-Verlag Berlin Heidelberg 2014

\begin{abstract}
Approval voting has attracted considerable attention in voting theory, but it has rarely been investigated in an Arrovian framework of collective preference ("social welfare") functions and never been connected with Arrow's impossibility theorem. The article explores these two directions. Assuming that voters have dichotomous preferences, it first characterizes approval voting in terms of its collective preference properties and then shows that these properties become incompatible if the collective preference is also taken to be dichotomous. As approval voting and majority voting happen to share the same collective preference function on the dichotomous domain, the positive result also bears on majority voting, and is seen to extend May's and Inada's early findings on this rule. The negative result is a novel and perhaps surprising version of Arrow's impossibility theorem, because the axiomatic inconsistency here stems from the collective preference range, not the individual preference domain.
\end{abstract}

The authors thank Claude d'Aspremont, Franz Dietrich, Jérôme Lang, Arunava Sen, Bill Zwicker, and two anonymous referees, for useful comments on an earlier version. The second author is grateful to CORE and the Australian National University for their hospitality when he was working on the paper.

F. Maniquet $(\varangle)$

CORE, Université Catholique de Louvain, Louvain-la-Neuve, Belgium

e-mail: francois.maniquet@uclouvain.be

F. Maniquet

Department of Economics, University of Warwick, Coventry, UK

P. Mongin

Centre National de la Recherche Scientifique, Paris, France

P. Mongin

HEC Paris, Paris, France

e-mail: mongin@greg-hec.com 


\section{Introduction}

Approval voting is the rule by which voters can cast votes for as many candidates as they wish, giving no more than one vote to each of them, and those candidates with the greatest vote total are elected. For the purpose of theoretical investigations, approval voting has been defined to be a social choice function, i.e., a mapping that associates a nonempty subset of candidates - the social choice - to every profile of individual characteristics - the individuals' preferences among candidates in one version, and their choices among them in another version. When it comes to voting generally, this choice-theoretic formalism has good descriptive value and it has the conceptual advantage of connecting well with strategic inquiries, including the landmark GibbardSatterthwaite theorem. Since Brams and Fishburn (1978) and Fishburn (1978a,b), it has been applied very effectively to approval voting. ${ }^{1}$

The present article departs from this tradition by defining approval voting as a social welfare function, or as we will rather call it to avoid irrelevant welfaristic suggestions, a collective preference function, that is to say, a mapping that associates a collective preference among candidates to every profile of individual preferences among these candidates. This formalism makes sense for a voting rule if the latter can be reinterpreted as an evaluation procedure, which is the case for approval voting. Here, the individuals would be asked to rank candidates into, say, "satisfactory" and "unsatisfactory", with their answers being pooled, but not necessarily followed by any actual collective decision (think of an opinion poll as against a real vote). To redescribe voting rules in this way is in fact an old idea of social choice theory, dating back to Arrow himself. He thought his original formalism to be applicable to them, and not only to welfare criteria, and accordingly introduced a collective preference function for majority voting (see 1963, ch. V). By taking the same step for approval voting, we shift the focus of its analysis from strategy-proofness to the related, but distinct condition of independence of irrelevant alternatives, as well as further Arrovian conditions such as positive responsiveness and the Pareto principle. To the best of our knowledge, Ju (2010) is the only scholar of approval voting who has already adopted a collective preference framework.

When approval voting is redefined in this way, it is natural to impose the restriction that individual preferences be dichotomous, i.e., have two indifference classes. Actually, since the early days, this restriction is also made in quite a few choice-theoretic analyses. $^{2}$ It will be upheld here, with two possibilities being investigated in turn: the

\footnotetext{
1 Fishburn (1978a,b) axiomatized approval voting in terms of a ballot aggregation function, which is an anonymous social choice function taking profiles of individual choices as its arguments. This framework is taken up in Sertel (1988), Alos-Ferrer (2006) and Sato (2014), and it inspires Baigent and Xu (1991) and Xu's (2010) related variants. The less formalistic, but pioneering paper by Brams and Fishburn (1978) emphasized the strategic properties of approval voting, and this line is recovered by Vorsatz $(2007 ; 2008)$ in a framework where the social choice function is defined on profiles of individual preferences, as in the Gibbard-Satterthwaite theorem. Some explicit body of game-theoretic analysis now exists (see Laslier and Sanver 2010). All this theoretical work is scant compared with the many practical studies on approval voting (see Brams and Fishburn 2005, and the relevant chapters in Laslier and Sanver 2010).

2 Brams and Fishburn (1978) emphasize that this case makes approval voting strategyproof, and Vorsatz (2007, 2008) assumes it throughout his analysis.
} 
simply dichotomous case, where only individual preferences are dichotomous, and the twice dichotomous case, where collective preferences also are. We present a characterization of approval voting in the former case, and one of dictatorship-thus an impossibility theorem-in the latter case. We may interpret the two results together as saying that the conditions for approval voting become inconsistent when the range of the collective preference function is subjected to the same dichotomous restriction as the domain. Our positive result relates to Ju's (2010) characterization, which also belongs to the simply dichotomous case; arguably, we go one step further into the Arrovian foundations of approval voting. As to our negative result, it is unprecedented, and it appears to be surprising compared with existing Arrovian impossibilities under restricted domains, because the logical ingredients of these impossibilities are missing here-in particular, the free triple property fails.

In the simply dichotomous case, the collective preference function of approval voting coincides with that of majority voting, as Arrow and followers have defined it. This observation connects our positive result with two classics, i.e., May's (1952) characterization of majority voting between two candidates, which we considerably extend, and Inada's (1964) proof that majority voting behaves well with dichotomous individual preferences, which we also recover and deepen (by replacing Inada's mere claim of possibility with a proper characterization). On the permissive dichotomous domain, majority voting can only be threatened by range restrictions, and this is what the negative result of the paper illustrates.

\section{Definitions and aggregative conditions}

As usual, a weak preference ordering $R$ on a set $X$ means a binary relation that is transitive, reflexive and complete; equivalently $R$ has an asymmetric part $P$ and a symmetric part $I$, which satisfy the $P P, P I, I P, I I$ variants of transitivity. The statements $x P y, x I y, x R y$ have their standard readings, i.e., " $x$ is strictly preferred to $y$ ", " $x$ is indifferent to $y$ ", " $x$ is strictly preferred or indifferent to $y$ ". An indifference class for $R$ is one of the equivalence classes generated by $I$, i.e., a set of the form $\left\{x \in X: x I x_{0}\right\}$ for some fixed $x_{0} \in X$.

As a particular case of $R$ on $X$, a dichotomous weak preference ordering has one or two non-empty indifference classes. It satisfies the $P P$ variant of transitivity vacuously and (for a sufficient number of elements) the $P I, I P$ and $I I$ variants non-vacuously. It can also be described in terms of its indifference classes directly. If $R$ has two indifference classes, we denote by $H$ and $L$ the higher and lower one, respectively, and if $R$ has only one indifference class, a case of complete indifference, we denote this class by CI. The obvious translation rules are:

$$
\begin{aligned}
x P y & \Leftrightarrow x \in H, y \in L \\
x I y & \Leftrightarrow \text { either } x, y \in H \text { or } x, y \in L \text { or } x, y \in C I .
\end{aligned}
$$

Define $\mathcal{O}$ to be the set of all weak preference orderings on $X$, and $\mathcal{D} \subset \mathcal{O}$ to be the set of dichotomous weak preference orderings on $X$. 
The technical literature sometimes defines dichotomous preferences to have exactly two non-empty indifference classes, thus excluding complete indifference (e.g., Brams and Fishburn 1978). We show below that our results extends to this stronger definition at a modest cost.

Given a set of candidates $X$ with cardinality $|X| \geq 3$ and a finite set of voters $N=\{1, \ldots, n\}$ with $n \geq 2$, we define a collective preference function $(C P F)$ to be a mapping

$$
F:\left(R_{1}, \ldots, R_{n}\right) \longmapsto R
$$

Throughout, the domain of $F$ will be dichotomous, with two cases considered in succession, i.e., $F: \mathcal{D}^{n} \rightarrow \mathcal{O}$ and $F: \mathcal{D}^{n} \rightarrow \mathcal{D}$; we call them the simply dichotomous and twice dichotomous case, respectively.

Correspondingly, the $C P F$ associated with approval voting can be stated in two different ways.

Notation 1 For any profile $\left(R_{1}, \ldots, R_{n}\right)$ in $\mathcal{O}^{n}$ or $\mathcal{D}^{n}$, we put $N\left(x P_{i} y\right)=\left\{i: x P_{i} y\right\}$ and $n\left(x P_{i} y\right)=\left|\left\{i: x P_{i} y\right\}\right|$ (similarly for $\left.R_{i}, I_{i}\right)$, and $N\left(x \in H_{i}\right)=\left\{i: x \in H_{i}\right\}$ and $n\left(x \in H_{i}\right)=\left|\left\{i: x \in H_{i}\right\}\right|$ (similarly for $\left.L_{i}, C I_{i}\right)$, and we put

$$
\operatorname{Max}\left(R_{1}, \ldots, R_{n}\right)=\left\{x \in X: n\left(x \in H_{i}\right) \geq n\left(y \in H_{i}\right), \forall y \in X\right\} .
$$

Definition $1 F$ is approval voting* if, for all $\left(R_{1}, \ldots, R_{n}\right) \in \mathcal{D}^{n}, x P y \Leftrightarrow n(x \in$ $\left.H_{i}\right)>n\left(y \in H_{i}\right)$.

Approval voting* trivially defines an ordering for any profile, and it has range $\mathcal{O}$, not $\mathcal{D}$, if $|X| \geq 3$. However, we may force the $\mathcal{D}$ range in redefining the $C P F$ thus.

Definition $2 \mathrm{~F}$ is approval voting** if, for every $\left(R_{1}, \ldots, R_{n}\right) \in \mathcal{D}^{n}$,

- if $\operatorname{Max}\left(R_{1}, \ldots, R_{n}\right) \neq X$, then

$$
H=\operatorname{Max}\left(R_{1}, \ldots, R_{n}\right) \text { and } L=X \backslash \operatorname{Max}\left(R_{1}, \ldots, R_{n}\right) ;
$$

- if $\operatorname{Max}\left(R_{1}, \ldots, R_{n}\right)=X$, then $R=C I$.

Under dichotomous individual preferences, majority voting has the same $C P F$ as approval voting*. Arrow $(1963$, p. 58$)$ defined the $C P F$ of majority voting by the condition that, for all $\left(R_{1}, \ldots, R_{n}\right)$ in the domain, $x R y$ if

$$
n\left(x R_{i} y\right) \geq n\left(y R_{i} x\right), \text { or equivalently } n\left(x P_{i} y\right) \geq n\left(y P_{i} x\right),
$$

and this definition has been endorsed by social choice theory. If the domain is $\mathcal{D}^{n}$ rather than $\mathcal{O}^{n}$, it collapses into that just given above-we skip the trivial argument. Since approval voting* always defines an ordering, we have by the same token proven Inada's (1964) classic observation that majority voting defines a transitive $C P F$ on the dichotomous domain.

Now to the properties that $C P F$ may satisfy. We begin by listing those which Arrow (1963) famously declared to be mutually inconsistent. 
Notation 2 For all $\left(R_{1}, \ldots, R_{n}\right),\left(R_{1}^{\prime}, \ldots, R_{n}^{\prime}\right)$ in $\mathcal{O}^{n}$ or $\mathcal{D}^{n}$, and all $x, y, z, w \in X$, we write $x R_{i} y \approx z R_{i}^{\prime} w$ instead of $\left(x R_{i} y \Leftrightarrow z R_{i}^{\prime} w\right.$ and $\left.y R_{i} x \Leftrightarrow w R_{i}^{\prime} z\right)$.

Condition 1 Independence of irrelevant alternatives (IIA): For all $\left(R_{1}, \ldots, R_{n}\right)$, $\left(R_{1}^{\prime}, \ldots, R_{n}^{\prime}\right) \in \mathcal{D}^{n}$ and all $x, y \in X$, if $x R_{i} y \approx x R_{i}^{\prime} y$ for all $i \in N$, then $x R y \Leftrightarrow$ $x R^{\prime} y$.

Condition 2 Weak Pareto (WP): For all $\left(R_{1}, \ldots, R_{n}\right) \in \mathcal{D}^{n}$ and all $x, y \in X$, if $x P_{i} y$ for all $i \in N$, then $x P y$.

Condition 3 Non-dictatorship (ND): There is no $j \in N$ such that for all $\left(R_{1}, \ldots, R_{n}\right) \in \mathcal{D}^{n}$ and all $x, y \in X$, if $x P_{j} y$, then $x P y$.

Both approval voting* and approval voting** satisfy $W P$. However, only the former, not the latter, satisfies IIA. The following 3-candidate, 2-individual profiles $\left(R_{1}, R_{2}\right)$, $\left(R_{1}^{\prime}, R_{2}^{\prime}\right)$ illustrates the failure: $x P_{1} y I_{1} z, z P_{2} y I_{2} x$ and $x I_{1}^{\prime} z P_{1}^{\prime} y, z P_{2}^{\prime} y I_{2}^{\prime} x$; by approval voting**, $x, z \in H, y \in L$, so $x P y$, and $z \in H^{\prime}, x, y \in L^{\prime}$, so $x I^{\prime} y$, contradicting IIA. Since approval voting* also satisfies $N D$, and then all of Arrow's conditions, his theorem indicates that the domain makes a difference, i.e., $\mathcal{D}^{n}$ does not behave like $\mathcal{O}^{n}$ (this is in effect Inada's observation). Arrow's theorem does not teach anything on approval voting ${ }^{* *}$ since this $C P F$ does not satisfy $I I A .^{3}$ The last section of the paper shows that this violation is forced by the range restriction.

\section{The collective preference function of approval voting (or majority voting)}

Here we characterize approval voting* (or equivalently majority voting) in terms of variant Arrovian conditions. First, this function obviously satisfies a vast strengthening of $N D$ :

Condition 4 Anonymity $(A)$ : For all $\left(R_{1}, \ldots, R_{n}\right) \in \mathcal{D}^{n}$ and all permutations $\sigma$ of $\{1, \ldots, n\}, F\left(R_{1}, \ldots, R_{n}\right)=F\left(R_{\sigma(1)}, \ldots, R_{\sigma(n)}\right)$.

Second, approval voting* satisfies Pareto conditions besides WP:

Condition 5 Pareto indifference $(P I)$ : For all $\left(R_{1}, \ldots, R_{n}\right) \in \mathcal{D}^{n}$ and all $x, y \in X$, if $x I_{i} y$ for all $i \in N$, then $x I y$.

Condition 6 Strict Pareto (SP): For all $\left(R_{1}, \ldots, R_{n}\right) \in \mathcal{D}^{n}$ and all $x, y \in X$, if $x R_{i} y$ for all $i \in N$ and $x P_{i} y$ for some $i$, then $x P y$.

It turns out that approval voting* can be characterized by adding IIA to these three conditions, or rather to the first two and $W P$, because this set will be shown to entail $S P$.

3 Approval voting does satisfy a weaker form of $I I A$, to wit: for all $\left(R_{1}, \ldots, R_{n}\right),\left(R_{1}^{\prime}, \ldots, R_{n}^{\prime}\right) \in \mathcal{D}^{n}$ and all $x, y \in X$, if $x R_{i} y \approx x R_{i}^{\prime} y$ for all $i \in N$, then $x P y \Longrightarrow x R^{\prime} y$. As Campbell and Kelly (2000) demonstrate after Baigent (1987), this Weak II A delivers a variant of Arrow's impossibility theorem in which $N D$ is replaced by a no-vetoer condition. 
Theorem 1 A collective preference function $F: \mathcal{D}^{n} \rightarrow \mathcal{O}$ is approval voting $*$ if and only if it satisfies IIA, A, PI, and WP.

The sufficiency part relies on three lemmas. The first derives a classic condition that strengthens IIA and is sometimes taken to be primitive by $C P F$ theory.

Condition 7 Neutrality $(N)$ : For all $\left(R_{1}, \ldots, R_{n}\right),\left(R_{1}^{\prime}, \ldots, R_{n}^{\prime}\right) \in \mathcal{D}^{n}$ and all $x, y, z, w \in X$, if $x R_{i} y \approx z R_{i}^{\prime} w$ for all $i \in N$, then $x R y \Leftrightarrow z R^{\prime} w$.

Lemma 1 If $F: \mathcal{D}^{n} \rightarrow \mathcal{O}$ satisfies IIA and PI, it satisfies $N$.

Proof Consider first the case of four distinct $x, y, z, w \in X$. By assumption, $\left(R_{1}, \ldots, R_{n}\right)$ and $\left(R_{1}^{\prime}, \ldots, R_{n}^{\prime}\right) \in \mathcal{D}^{n}$ are s.t. $x R_{i} y \approx z R_{i}^{\prime} w$ for all $i \in N$. Take $\left(\bar{R}_{1}, \ldots, \bar{R}_{n}\right) \in \mathcal{D}^{n}$ s.t. $x R_{i} y \approx x \bar{R}_{i} y$ for all $i \in N$, and s.t. $x \bar{I}_{i} z$ and $y \bar{I}_{i} w$ for all $i \in N$. Thus, by construction, $z \bar{R}_{i} w \approx z R_{i}^{\prime} w$ for all $i \in N$. Suppose that $x R y$. Then, $x \bar{R} y$ follows from IIA, $z \bar{R} w$ from $P I$, and finally $z R^{\prime} w$ from IIA.

Related proofs take care of the two cases in which there are three distinct elements among $x, y, z, w \in X, x \neq y$, and the position of the common element is the same in the two pairs, i.e., $x=z$ or $y=w$. Now, suppose that the common element changes position, i.e., $x=w$ or $y=z$. We give a proof for the former case. By assumption, $\left(R_{1}, \ldots, R_{n}\right)$ and $\left(R_{1}^{\prime}, \ldots, R_{n}^{\prime}\right) \in \mathcal{D}^{n}$ are s.t. $x R_{i} y \approx z R_{i}^{\prime} x$ for all $i \in N$. Take $\left(\bar{R}_{1}, \ldots, \bar{R}_{n}\right) \in \mathcal{D}^{n}$ s.t. $x R_{i} y \approx z \bar{R}_{i} y$ for all $i \in N$. From one of the cases with unchanged positions, $x R y \Leftrightarrow z \bar{R} y$. By construction, $z R_{i}^{\prime} x \approx z \bar{R}_{i} y$ for all $i \in N$, so from the other case, $z R^{\prime} x \Leftrightarrow z \bar{R} y$, and finally $x R y \Leftrightarrow z R^{\prime} x$.

If there are three distinct elements among $x, y, z, w \in X$, and $x=y$, or $z=w, N$ reduces to $P I$.

If there are two distinct elements, say $x$ and $y$, which do not exchange positions, $N$ reduces to IIA. Otherwise, suppose that $\left(R_{1}, \ldots, R_{n}\right)$ and $\left(R_{1}^{\prime}, \ldots, R_{n}^{\prime}\right) \in \mathcal{D}^{n}$ are s.t. $x R_{i} y \approx y R_{i}^{\prime} x$ for all $i \in N$. Take $z \neq x, y$ and $\left(\bar{R}_{1}, \ldots, \bar{R}_{n}\right) \in \mathcal{D}^{n}$ s.t. $x R_{i} y \approx x \bar{R}_{i} z$ for all $i \in N$. It follows that $x R y \Leftrightarrow x \bar{R} z$. Take $\left(\overline{\bar{R}}_{1}, \ldots, \overline{\bar{R}}_{n}\right) \in \mathcal{D}^{n}$ s.t. $y R_{i}^{\prime} x \approx y \overline{\bar{R}}_{i} z$ for all $i \in N$. It follows that $y R^{\prime} x \Leftrightarrow y \overline{\bar{R}} z$. Now, by construction, $x \bar{R}_{i} z \approx y \overline{\bar{R}}_{i} z$ for all $i \in N$, whence $x \bar{R} z \Leftrightarrow y \overline{\bar{R}} z$. Combining the equivalences, one gets $x R y \Leftrightarrow y R^{\prime} x$, as desired.

The second lemma derives another condition that is sometimes also taken to be primitive. In preparation for the next section, we give it in two variants, the former being weaker than the latter. They are distinguished only by their consequent clauses.

Condition 8 Positive responsiveness 1 (PRl): For all $\left(R_{1}, \ldots, R_{n}\right),\left(R_{1}^{\prime}, \ldots, R_{n}^{\prime}\right) \in$ $\mathcal{D}^{n}$ and all $x, y \in X$, if $x P_{i} y \Rightarrow x P_{i}^{\prime} y$ and $x I_{i} y \Rightarrow x R_{i}^{\prime} y$ for all $i \in N$, and $y P_{j} x$ and $x R_{j}^{\prime} y$, or $x I_{j} y$ and $x P_{j}^{\prime} y$, for some $j \in N$, then $x P y \Rightarrow x P^{\prime} y$.

Condition 9 Positive responsiveness 2 (PR2): For all $\left(R_{1}, \ldots, R_{n}\right),\left(R_{1}^{\prime}, \ldots, R_{n}^{\prime}\right) \in$ $\mathcal{D}^{n}$ and all $x, y \in X$, if $x P_{i} y \Rightarrow x P_{i}^{\prime} y$ and $x I_{i} y \Rightarrow x R_{i}^{\prime} y$ for all $i \in N$, and $y P_{j} x$ and $x R_{j}^{\prime} y$, or $x I_{j} y$ and $x P_{j}^{\prime} y$, for some $j \in N$, then $x R y \Rightarrow x P^{\prime} y$.

Lemma 2 If $F: \mathcal{D}^{n} \rightarrow \mathcal{O}$ satisfies $N$ and $S P$, it satisfies $P R 2$. 
Proof To derive $P R 2$, we first assume that $\left(R_{1}, \ldots, R_{n}\right),\left(R_{1}^{\prime}, \ldots, R_{n}^{\prime}\right) \in \mathcal{D}^{n}$ and $x, y \in X$ meet the antecedent condition without any full reversal of strict preference, i.e., without any $j$ s.t. $y P_{j} x$ and $x P_{j}^{\prime} y$.

Take $z \neq x, y$ and $\left(\bar{R}_{1}, \ldots, \bar{R}_{n}\right) \in \mathcal{D}^{n}$ so defined: for all $i \in N$,

- if $x P_{i} y$ and $x P_{i}^{\prime} y$, then $x \bar{P}_{i} y \bar{I}_{i} z$; if $y P_{i} x$ and $y P_{i}^{\prime} x$, then $z \bar{I}_{i} y \bar{P}_{i} x$; if $x I_{i} y$ and $x I_{i}^{\prime} y$, then $x \bar{I}_{i} y \bar{I}_{i} z$

- if $y P_{i} x$ and $x I_{i}^{\prime} y$, then $z \bar{P}_{i} x \bar{I}_{i} y$; if $x I_{i} y$ and $x P_{i}^{\prime} y$, then $z \bar{I}_{i} x \bar{P}_{i} y$.

Thus, for all $i \in N, x R_{i} y \approx x \bar{R}_{i} z, z \bar{R}_{i} y$, and $x R_{i}^{\prime} y \approx x \bar{R}_{i} y$. We also note by inspecting the possibilities that for some $i, z \bar{P}_{i} y$. Now, suppose that $x R y$. Then, $x \bar{R} z$ by $N$, and because $S P$ entails that $z \bar{P} y$, it follows that $x \bar{P} y$, hence $x P^{\prime} y$ by IIA. This completes the proof of $P R 2$ in the case just considered.

If $\left(R_{1}, \ldots, R_{n}\right),\left(R_{1}^{\prime}, \ldots, R_{n}^{\prime}\right) \in \mathcal{D}^{n}$ and $x, y \in X$ meet the antecedent of $P R 2$ in full generality, take $\left(R_{1}^{\prime \prime}, \ldots, R_{n}^{\prime \prime}\right) \in \mathcal{D}^{n}$ s.t., for all $i \in X, x I_{i}^{\prime \prime} y$ if $y P_{i} x$ and $x P_{i}^{\prime} y$, and $x R_{i}^{\prime \prime} y \approx x R_{i}^{\prime} y$ otherwise. Suppose that $x R y$. By the case just proved, $x P^{\prime \prime} y$, and again by this case, $x P^{\prime} y$.

The third lemma shows that $S P$ can be replaced by $W P$ in the characterization of approval voting*.

Lemma 3 If $F: \mathcal{D}^{n} \rightarrow \mathcal{O}$ satisfies $N$, WP and A, it satisfies $S P$.

Proof Fix $x, y$ and a sequence of profiles $\left(R_{1}^{k}, \ldots, R_{n}^{k}\right) \in \mathcal{D}^{n}, k \in\{1, \ldots, n\}$, s.t.

$$
x P_{i}^{k} y, 1 \leq i \leq k \text { and } x I_{i}^{k} y, k+1 \leq i \leq n .
$$

In view of $N$ and $A$, it is sufficient to show that $S P$ holds for this pair and this sequence (note that $k=n$ corresponds to $W P$ ). The proof goes by induction on $k$.

- $k=1$. Suppose that $y R^{1} x$; we reach a contradiction with $W P$ by showing that $y R^{l} x$ holds for all $l \in\{1, \ldots, n\}$, hence in particular for $l=n$. The initial supposition covers the case $l=1$; now suppose we have proved that $y R^{l} x$ for some $l \in\{1, \ldots, n-1\}$. Let us take the profiles $\left(R_{1}^{l+1}, \ldots, R_{n}^{l+1}\right) \in \mathcal{D}^{n}$, and for some $z \neq x, y,\left(\bar{R}_{1}^{l+1}, \ldots, \bar{R}_{n}^{l+1}\right) \in \mathcal{D}^{n}$ s.t.

$$
\begin{aligned}
x \bar{R}_{i}^{l+1} y & \approx x R_{i}^{l+1} y \text { for all } i \in N, \\
z \bar{I}_{i}^{l+1} y \text { for all } i & \neq l+1 \text { and } z \bar{I}_{l+1}^{l+1} x \bar{P}_{l+1}^{l+1} y .
\end{aligned}
$$

Then, we apply $N$ in a comparison with $\left(R_{1}^{l}, \ldots, R_{n}^{l}\right)$, and $N$ and $A$ in a comparison with $\left(R_{1}^{1}, \ldots, R_{n}^{1}\right)$, to get $z \bar{R}^{l+1} x$ and $y \bar{R}^{l+1} z$, hence $y \bar{R}^{l+1} x$. So we have proved by induction that $y R^{l} x$ holds for all $l \in\{1, \ldots, n\}$, as desired.

- Suppose $S P$ holds for $\left(R_{1}^{k}, \ldots, R_{n}^{k}\right)$ and consider $\left(R_{1}^{k+1}, \ldots, R_{n}^{k+1}\right)$. In the previous definition of $\left(\bar{R}_{1}^{l+1}, \ldots, \bar{R}_{n}^{l+1}\right)$, replace $l$ by $k$; this defines a profile $\left(\bar{R}_{1}^{k+1}, \ldots, \bar{R}_{n}^{k+1}\right)$ which, by comparison with $\left(\bar{R}_{1}^{k}, \ldots, \bar{R}_{n}^{k}\right)$, satisfies $x \bar{P}^{k+1} z$ by $S P$, and by comparison with $\left(\bar{R}_{1}^{1}, \ldots, \bar{R}_{n}^{1}\right)$, satisfies $z \bar{P}^{k+1}$ y by $A$ and $S P$. Hence $x \bar{P}^{k+1} y$, as was to be proved. 
Proof (Theorem 1) Suppose that there is some $F: \mathcal{D}^{n} \rightarrow \mathcal{O}$ that is not approval voting*. Then, there are $\left(R_{1}, \ldots, R_{n}\right) \in \mathcal{D}^{n}$ and $x, y \in X$ s.t. either (i) $n\left(x \in H_{i}\right)=$ $n\left(y \in H_{i}\right)$ and $x P y$, or (ii) $n\left(x \in H_{i}\right)>n\left(y \in H_{i}\right)$ and $y R x$.

In case (i), there are three groups of individuals, i.e., $N\left(x P_{i} y\right), N\left(y P_{i} x\right), N\left(x I_{i} y\right)$ with $n\left(x P_{i} y\right)=n\left(y P_{i} x\right)$. The first two groups are non-empty by $P I$. We may take a permutation $\sigma$ that interchanges them and leaves the third group unchanged; by $A$, the resulting profile $\left(R_{\sigma(1)}, \ldots, R_{\sigma(n)}\right)$ has the collective preference $x P_{\sigma} y$. Now, observing that for all $i \in N, x R_{i} y \approx y R_{\sigma(i)} x$, we apply $N$ to the profile to get the contradiction that $y P_{\sigma} x$.

In case (ii), the three groups of individuals $N\left(x P_{i} y\right), N\left(y P_{i} x\right), N\left(x I_{i} y\right)$ are s.t. $n_{1}=n\left(x P_{i} y\right)>n_{2}=n\left(y P_{i} x\right)$. The second group is non-empty by $S P$, and from the inequality, the first group also is. Take a permutation $\sigma$ that interchanges $n_{2}$ individuals in $N\left(x P_{i} y\right)$ with those in $N\left(y P_{i} x\right)$ and leaves the position of any others unchanged; by $A$, the resulting profile $\left(R_{\sigma(1)}, \ldots, R_{\sigma(n)}\right)$ has the collective preference $y R_{\sigma} x$. Now, modify this profile into $\left(R_{1}^{\prime}, \ldots, R_{n}^{\prime}\right)$ by putting $y P_{i}^{\prime} x$ if $i=\sigma(i)$ is any of the remaining $n_{1}-n_{2}$ individuals of $N\left(x P_{i} y\right)$ and leaving any other individual's preference the same. Given this reinforcement of strict preference for $y, P R 2$ entails that $y P^{\prime} x$. However, $\left(R_{1}^{\prime}, \ldots, R_{n}^{\prime}\right)$ also modifies $\left(R_{1}, \ldots, R_{n}\right)$ in such a way that $N$ entails that $x R^{\prime} y$, a contradiction.

Let us briefly check the logical independence of each condition in Theorem 1 . That $P I$ is independent of the others can be seen by revising the $C P F$ of approval voting* so as to break ties between $x$ and $y$ when $n\left(x \in H_{i}\right)=n\left(y \in H_{i}\right)$; such a function will satisfy all conditions of approval voting* except for PI. For example, one may single out $\bar{x}, \bar{y} \in X$ and decide that any tie between either $\bar{x}$ or $\bar{y}$ and $z \neq \bar{x}, \bar{y}$ is broken in favour of $z$, while ties between $\bar{x}$ and $\bar{y}$ are broken thus: $\bar{x}$ prevails if the equal number of supporters is even, and $\bar{y}$ prevails if it is odd; any other tie occurring in $X$ is left unbroken. Not every $C P F$ that satisfies all conditions but $P I$ reduces to a tie-breaking revision of approval voting*. ${ }^{4}$ Fix a function $w$ from $X$ to $[0, n-1]$, with the $w(x)$ not all equal, and replace approval voting* by the following $C P F$ : for all $\left(R_{1}, \ldots, R_{n}\right) \in \mathcal{D}^{n}$

$$
x P y \Leftrightarrow n\left(x \in H_{i}\right)+w(x)>n\left(y \in H_{i}\right)+w(y) .
$$

This $C P F$ obviously satisfies $I I A$ and $A$, and obviously violates $P I$; it satisfies WP by the range condition on $w$. It differs from the previous class of examples because, for suitable numerical values, it can violate even the strict preference part of approval voting* and it can create ties that this $C P F$ does not have. The conceptual dissimilarity between the two classes suggests that very little structure is left when the only conditions are IIA, $A$ and WP. We eschew the problematic characterization task in this paper.

Similarly, that IIA is independent of the other conditions follows from considering the $C P F$ that corresponds to equal cumulative voting, which is another refinement of approval voting: each voter has one vote and divides it evenly among the candidates

$\overline{4}$ The following example adapts a suggestion made by Bill Zwicker. 
he approves of; candidates with the greatest total of fractional votes are elected. The independence of $A$ results from considering dictatorial $C P F$.

Inspection of the proofs shows that the complete indifference ordering $C I \in \mathcal{D}$ does not occur as an auxiliary profile unless $|X|=3$ (see the relevant step in the proof of Lemma 2). Thus, denoting $\mathcal{D}^{-}=\mathcal{D} \backslash\{C I\}$, we have the following extension of the theorem.

Remark 1 If $|X| \geq 4$, the theorem also holds for $F:\left(\mathcal{D}^{-}\right)^{n} \rightarrow \mathcal{D}$.

With this extension at hand, we can redefine dichotomous preferences to have exactly two indifference classes, as in some of the other work on approval voting.

To the best of our knowledge, only Ju (2010) has characterized approval voting in terms of $C P F .^{5}$ Assuming the simply dichotomous framework, he shows that plurality voting, hence implicitly approval voting*, is that $C P F$ which satisfies $A, N$, plus a positive responsiveness and a non-trivality condition, here denoted as $P R 3$ and $N T$, which we now define.

Condition 10 Positive responsiveness 3(PR3): For all $\left(R_{1}, \ldots, R_{n}\right),\left(R_{1}^{\prime}, \ldots, R_{n}^{\prime}\right) \in$ $\mathcal{D}^{n}$ and all $x, y \in X$, if $x P_{i} y \Rightarrow x P_{i}^{\prime} y$ and $x I_{i} y \Rightarrow x R_{i}^{\prime} y$ for all $i \in N$, and $y P_{i} x$ and $x R_{i}^{\prime} y$, or $x I_{i} y$ and $x P_{i}^{\prime} y$, for some $i$, then $x R y \Rightarrow x R^{\prime} y$.

Condition 11 Non-triviality (NT): F does not have the constant value CI.

$P R 3$ is weaker than $P R 2$ and by itself incomparable with $P R 1$. However, we can obtain Ju's characterization from ours by an added step of reasoning. Since $N$ entails $I I A$ and $P I$, it is sufficient to show that $N, P R 3$, and $N T$ entail $W P$, and we will have achieved the desired logical connection.

Corollary 1 (Ju 2010) A collective preference function $F: \mathcal{D}^{n} \rightarrow \mathcal{O}$ is approval voting* if and only if it satisfies $N, A, P R 3$ and NT.

Proof To be shown that WP holds. Given $\left(R_{1}, \ldots, R_{n}\right) \in \mathcal{D}^{n}$ and $x, y \in X$ satisfying $x P_{i} y, i \in N$, take $z \neq x, y,\left(R_{1}^{\prime}, \ldots, R_{n}^{\prime}\right) \in \mathcal{D}^{n}$ s.t. $z I_{i}^{\prime} x P_{i}^{\prime} y, i \in N$, and $\left(R_{1}^{\prime \prime}, \ldots, R_{n}^{\prime \prime}\right) \in \mathcal{D}^{n}$ s.t. $z P_{i}^{\prime \prime} x I_{i}^{\prime \prime} y, i \in N . N$ (or $P I$ ) leads to $x I^{\prime \prime} y, P R 3$ to $x R^{\prime} y$, and IIA to $x R y$. It remains to exclude that $x I y$. If this indifference holds, we can interchange $x$ and $y$ in $\left(R_{1}, \ldots, R_{n}\right)$ and obtain $\left(\bar{R}_{1}, \ldots, \bar{R}_{n}\right) \in \mathcal{D}^{n}$ with $y \bar{P}_{i} x$, $i \in N$, and thus $y \bar{I} x$ by $N$. Now, take any profile whatever: by an application of $P R 3$ based on either $\left(R_{1}, \ldots, R_{n}\right)$ or $\left(\bar{R}_{1}, \ldots, \bar{R}_{n}\right)$, it follows that $x$ and $y$ are collectively indifferent. This would hold for any choice of $x, y \in X$, hence violate $N T$.

Conversely, one may recover our characterization from Ju's once Lemmas 1 and 2 have been established-his theorem then playing the role of Lemma 3 and the End of Proof. As this further comparison suggests, the two equivalent sets of conditions for approval voting* are not quite at the same axiomatic level. In essence, by adding Lemmas 1 and 2, we replace $N$ by the more Arrovian IIA condition, plus PI, and we reformulate $P R$ the way it should be when this change it made.

5 Essentially the same paper appears as Ju (2011). 
As already said, the other available characterizations of approval voting are choicetheoretic, hence not directly comparable with ours. However, Vorsatz (2007) defines social choice functions on profiles of dichotomous individual preferences-our simply dichotomous case - and characterizes the social choice function of approval voting* in terms of four conditions, i.e., Anonymity, Neutrality, Strategyproofness and Strict monotonicity, which are reminiscent of ours. Neutrality is like a much weaker form of $N$, Strict monotonicity suggests $P R 3$, and IIA holds in effect, as Vorsatz derives its choice-theoretic version from Neutrality and Strategyproofness. By contrast, following Fishburn (1978a, b), Sato (2014) defines social choice functions on profiles individual choices rather than of dichotomous preferences. Thus, his characterization in terms of Anonymity, Neutrality and Faithfulness (another monotonicity condition) is more remote. $^{6}$

If we interpret our $C P F$ in terms of majority voting, as the domain permits, we have a further connection with May's (1952) classic result on majority voting between two candidates. Expanding on Arrow's theorem for this special case, May characterized the $C P F$ of majority voting by three conditions, which are $N, A$ and $P R 2$ when translated into the present framework. This is but a modest corollary to Theorem 1. As the latter makes clear, the relevant cardinality restriction bears on the voters' sets of equivalence classes, and not on the set of candidates. Concerning Inada's (1964) observation, we have extended it by fully characterizing the $C P F$ of majority voting on the dichotomous domain, instead of simply noting that it satisfies Arrow's ordering condition on this domain.

\section{From approval voting to dictatorship}

In this section, we shift to the twice dichotomous case and demonstrate that the conditions characterizing approval voting* in the simply dichotomous case now characterize dictatorship. We actually prove the more powerful result that Arrow's theorem with its initial conditions holds in the twice dichotomous case.

Theorem 2 No collective preference function $F: \mathcal{D}^{n} \rightarrow \mathcal{D}$ satisfies IIA, WP and ND.

The proof goes through four lemmas.

Condition 12 Pareto Preference (PP): For all $\left(R_{1}, \ldots, R_{n}\right) \in \mathcal{D}^{n}$ and all $x, y \in X$, if $x R_{i} y$ for all $i \in N$, then $x R y$.

Lemma 4 If a collective preference function $F: \mathcal{D}^{n} \rightarrow \mathcal{D}$ satisfies IIA and WP, it satisfies PI and PP.

Proof Consider $\left(R_{1}, \ldots, R_{n}\right) \in \mathcal{D}^{n}$ and $x, y \in X$ s.t. $x R_{i} y$ for all $i \in N$. Take $z \neq x, y$ and $\left(R_{1}^{\prime}, \ldots, R_{n}^{\prime}\right) \in \mathcal{D}^{n}$ s.t. $x P_{i}^{\prime} z$ and $x R_{i} y \approx x R_{i}^{\prime} y$. (If $x P_{i} y, y \in L_{i}^{\prime}$ and if

\footnotetext{
6 Both Vorsatz (2007) and Sato (2014) actually have more conditions. They allow for a varying set of candidates $X$ and-following Fishburn (1978a, b) — for a variable finite set of individuals $N$. This leads them to introduce consistency conditions to connect the social choice functions obtained for these diverse sets.
} 
$x I_{i} y, y \in H_{i}^{\prime}$ ). If $x I_{i} y$ for all $i \in N$, then $x I_{i}^{\prime} y$ for all $i \in N$. In this case, $W P$ entails that $x P^{\prime} z$ and $y P^{\prime} z$, so $x I^{\prime} y$ since $F$ has range $\mathcal{D}$, and $x I y$ by IIA. This completes the derivation of $P I$. In the general case where $x R_{i} y$ for all $i \in N, W P$ entails that $x P^{\prime} z$, and the $\mathcal{D}$ range that $x R^{\prime} y$, whence $x R y$ by IIA. This completes the derivation of $P P$.

Lemma 5 If a collective preference function $F: \mathcal{D}^{n} \rightarrow \mathcal{D}$ satisfies IIA and PI, it satisfies $N$.

Proof Same as for Lemma 1. (The $\mathcal{D}$ range plays no role in this proof).

Lemma 6 If a collective preference function $F: \mathcal{D}^{n} \rightarrow \mathcal{D}$ satisfies $N$ and WP, it satisfies PR1.

Proof As in the proof of Lemma 2, we begin by assuming that $\left(R_{1}, \ldots, R_{n}\right)$, $\left(R_{1}^{\prime}, \ldots, R_{n}^{\prime}\right) \in \mathcal{D}^{n}$ and $x, y \in X$ meet the antecedent condition without any full reversal of strict preference. With $z \neq x, y$ and $\left(\bar{R}_{1}, \ldots, \bar{R}_{n}\right) \in \mathcal{D}^{n}$ as defined in this proof, we have again that, for all $i \in N, x R_{i} y \approx x \bar{R}_{i} z, z \bar{R}_{i} y$, and $x R_{i}^{\prime} y \approx x \bar{R}_{i} y$. Since $N$ implies IIA, $P P$ holds by Lemma 4, hence $z \bar{R} y$. Now if $x P y$, then $x \bar{P} z$ by $N$, and from the last fact, $x \bar{P} y$, whence $x P^{\prime} y$ by IIA. The end of the proof parallels that of Lemma 2.

A group $M \subseteq N$ is said to be decisive if for all pairs $(x, y) \in X^{2}$ and all profiles $\left(R_{1}, \ldots, R_{n}\right) \in \mathcal{D}^{n}$,

$$
x P_{i} y, i \in M \Longrightarrow x P y,
$$

and it is said to be semi-decisive on the pair $(x, y) \in X^{2}$ in the profile $\left(R_{1}, \ldots, R_{n}\right) \in$ $\mathcal{D}^{n}$ if

$$
x P_{i} y, i \in M, y R_{i} x, i \in N \backslash M \text {, and } x P y .
$$

Lemma 7 If a collective preference function $F: \mathcal{D}^{n} \rightarrow \mathcal{D}$ satisfies $N$ and PRl, any group $M \subseteq N$ that is semi-decisive on a pair $(x, y) \in X^{2}$ in a profile $\left(R_{1}, \ldots, R_{n}\right) \in$ $\mathcal{D}^{n}$ is decisive.

Proof Take $M$ as specified, partition $N \backslash M$ into $G_{1}=\left\{i \mid y P_{i} x\right\}$ and $G_{2}=\left\{i \mid y I_{i} x\right\}$, and consider the profiles $\left(R_{1}^{\prime}, \ldots, R_{n}^{\prime}\right) \in \mathcal{D}^{n}$ s.t.

$$
x P_{i}^{\prime} y, i \in M, y P_{i}^{\prime} x, i \in G_{1} \text {, and } y P_{i}^{\prime} x \text { for at least one } i \in G_{2} \text {. }
$$

If we show that $M$ is semi-decisive on the given $(x, y)$ for all such profiles $\left(R_{1}^{\prime}, \ldots, R_{n}^{\prime}\right)$, the conclusion that $M$ is decisive will follow by $P R 1$ and $N$.

To establish the desired property that $x P^{\prime} y$, take $z \neq x, y$ and $\left(R_{1}^{\prime \prime}, \ldots, R_{n}^{\prime \prime}\right)$ s.t. for all $i \in N, z R_{i}^{\prime \prime} y \approx x R_{i} y$ and $x R_{i}^{\prime \prime} y \approx x R_{i}^{\prime} y . N$ entails that $z P^{\prime \prime} y$ and would entail $y P^{\prime \prime} x$ if $y P^{\prime} x$ held, but this is prohibited by the $\mathcal{D}$ range. Hence $x R^{\prime} y$. It remains to show that $x I^{\prime} y$ is impossible. 
If $x I^{\prime} y$, then $x I^{\prime \prime} y$ by $N$, and $z P^{\prime \prime} x$ follows. From $I I A$, any profile $\left(R_{1}^{\prime \prime \prime}, \ldots, R_{n}^{\prime \prime \prime}\right) \in$ $\mathcal{D}^{n}$ s.t. for all $i \in N, z R_{i}^{\prime \prime \prime} x \approx z R_{i}^{\prime \prime} x$ satisfies $z P^{\prime \prime \prime} x$. The $\mathcal{D}^{n}$ domain contains such a profile with $x R_{i}^{\prime \prime \prime} y \approx x R_{i} y$ for all $i \in N$. (For a proof, see the construction:

- $x, z \in H_{i}^{\prime \prime \prime}$ and $y \in L_{i}^{\prime \prime \prime}$ if $i \in M$;

- $y \in H_{i}^{\prime \prime \prime}$ and $x, z \in L_{i}^{\prime \prime \prime}$ if $i \in G_{1}$;

- $x I_{i}^{\prime \prime \prime} y I_{i}^{\prime \prime \prime} z$ if $i \in G_{2}$ and $x I_{i}^{\prime} y$;

- $z \in H_{i}^{\prime \prime \prime}$ and $x, y \in L_{i}^{\prime \prime \prime}$ if $i \in G_{2}$ and $y P_{i}^{\prime} x$.

Now, IIA entails $x P^{\prime \prime \prime} y$, which is impossible given the $\mathcal{D}$ range. This completes the proof that $x P^{\prime} y$.

Define $V \subseteq 2^{N}$ to be the set of all decisive groups. To contradict $N D$ is tantamount to showing that $V$ contains a singleton, and we proceed to this last stage of the argument.

Proof (Theorem 2) The set $V$ is non-empty because $N \in V$ in virtue of $W P$. Since $N$ is finite, there exists in $V$ a group of smallest cardinality $M^{*}$, which cannot be $\varnothing$ in virtue of the $P P$ property secured by Lemma 4 . With Lemmas 5 and 6 at hand, the conclusion of Lemma 7 follows. We use it to show that $M^{*}$ is a singleton.

Suppose by way of contradiction that $\left|M^{*}\right| \geq 2$, so that $M^{*}$ can be partitioned into two non-empty groups $M_{1}^{*}, M_{2}^{*}$. We take $x, y, z \in X$ and a profile $\left(R_{1}, \ldots, R_{n}\right) \in \mathcal{D}^{n}$ with the following properties:

- for all $i \in M_{1}^{*}, x P_{i} y I_{i} z$

- for all $i \in M_{2}^{*}, z I_{i} x P_{i} y$;

- for all $i \in N \backslash M^{*}, y P_{i} x I_{i} z$.

It follows, first, that $x P y$ because $M^{*}=M_{1}^{*} \cup M_{2}^{*}$ is decisive, and second, that $y R z$ because $z P y$ would mean that $M_{2}^{*}$ is semi-decisive on $(z, y)$ in $\left(R_{1}, \ldots, R_{n}\right)$, hence decisive, and this would contradict the minimality of $M^{*}$. The two conclusions entail that $x P z$, but this would mean that $M_{1}^{*}$ is semi-decisive on $(x, z)$ in $\left(R_{1}, \ldots, R_{n}\right)$, hence decisive, another contradiction with the minimality of $M^{*}$.

Like Arrow's theorem for $F: \mathcal{O}^{n} \rightarrow \mathcal{O}$, Theorem 2 says that $F: \mathcal{D}^{n} \rightarrow \mathcal{D}$ satisfying IIA and WP is a dictatorship, i.e., there is $j \in N$-the dictator-such that, for all $\left(R_{1}, \ldots, R_{n}\right)$ in the respective domain and for all $x, y \in X$, if $x P_{j} y$, then $x P y$. However, more is true with the present $F$ than with Arrow's. By the same argument that proves $P I$ in Lemma $4, j$ also dictates collective indifference, i.e., for all $\left(R_{1}, \ldots, R_{n}\right) \in \mathcal{D}^{n}$ and all $x, y \in X, x P_{j} y$ if and only if $x P y$. It follows that $S P$ cannot hold with the present $F$, this unusual restriction being due to its range. Consistently, the proof of Lemma 3, which derives SP for $F: \mathcal{D}^{n} \rightarrow \mathcal{O}$, made use of at least three indifference classes.

Inspection of the present proof (see Lemmas 6 and 7) shows that Remark 1 also applies to Theorem 2.

The formal argument has gone through the three stages of neutrality, positive responsiveness, and finally dictatorship, which are familiar from early proofs in social choice theory, but dichotomous individual preferences do not support arguments that make active use of $P P$-transitivity, so each stage needed a special proof based on either the domain $\mathcal{D}^{n}$ or both this domain and the range $\mathcal{D}$ (in Lemmas 4 and 7). Relatedly, 
there is no use here for the free triple property, which is the main sufficient condition on individual preference domains to derive an Arrovian impossibility. Social choice theory rarely, if ever, considers restrictions put on both the domain and range of the $C P F$; see the surveys by Gaertner (2002) and Breton and Weymark (2011).

At virtually each stage, we made flexible use of the voters' indifference relation, i.e., we interpreted $x I_{i} y$ as being sometimes $x, y \in H_{i}$, sometimes $x, y \in L_{i}$, depending on what was to be proved. The dictatorial conclusion would collapse without this convenience. To illustrate, consider the subset $\mathcal{D}^{S}$ of those weak dichotomous orderings the higher class of which is a singleton. There exist non-dictatorial $F:\left(\mathcal{D}^{S}\right)^{n} \rightarrow \mathcal{D}$ satisfying both $I I A$ and $W P$, e.g., the following $C P F$ : for all $\left(R_{1}, \ldots, R_{n}\right) \in \mathcal{D}^{S}$, if $x P_{i} y$ for all $i \in N$, then $x P y$; otherwise,

$$
F\left(R_{1}, \ldots, R_{n}\right)=C I \text {. }
$$

However, dictatorhip would be reinstated if the conditions applied to $F:\left(\mathcal{D}^{S}\right)^{n} \rightarrow$ $\mathcal{D}^{S}$. We do not prove this further Arrovian variant here.

A final comment is in order. One may wonder how to place Theorem 2 vis-à-vis the current analysis of approval voting in terms of social choice functions. The answer is that it has no analogue in this framework. Superficially, dichotomous preferences resemble choices, since both divide the set of candidates into a higher and a lower subset. However, an expressed choice can result from preferences having more than two indifference sets, so that indifference within the higher subset and within the lower subset cannot be presumed in the case of choice, whereas the $H$ and $L$ of a dichotomous preference are indifference sets by construction. Thus, the formalism of Theorem 2 is much more assertive than a choice-theoretic one could ever be. This holds even when the social choice function is defined on dichotomous individual preferences, since the gap between preference and choice remains at the collective level. In sum, although the choice-theoretic and the preference-theoretic formalisms are both suited for positive characterizations of approval voting, only the former can reach the critical level of Theorem 2 .

\section{References}

Alos-Ferrer C (2006) A simple characterization of approval voting. Soc Choice Welfare 27:621-625

Arrow KJ (1963) Social choice and individual values, 2nd ed. Yale University Press, New Haven (1st ed., 1951)

Baigent N (1987) Twitching weak dictators. J Econ 47:407-411

Baigent N, Xu Y (1991) Independent necessary and sufficient conditions for approval voting. Math Soc Sci 21:21-29

Brams SJ, Fishburn PC (1978) Approval voting. Am Polit Sci Rev 72:831-847

Brams SJ, Fishburn PC (2005) Going from theory to practice: the mixed success of approval voting. Soc Choice Welfare 25:457-474

Campbell DE, Kelly JS (2000) Weak independence and veto power. Econ Lett 66:183-189

Fishburn PC (1978a) Symmetric and consistent aggregation with dichotomous preferences. In: Laffont J (ed) Aggreg Revel Prefer. Elsevier, Amsterdam

Fishburn PC (1978b) Axioms for approval voting: direct proof. J Econ Theory 19:180-185

Gaertner W (2002) Domain restrictions. In: Arrow KJ, Sen AK, Suzumura K (eds) Handbook of social choice and welfare. Amsterdam, North Holland, I, Chap 3, pp 131-170 
Inada K (1964) A note on the simple majority decision rule. Econometrica 32:525-531

Ju BG (2010) "Collective choice for simple preferences. In: Laslier JF, Sanver MR (eds) Handbook of approval voting. Springer, New York, Chap 4, pp 41-90

Ju BG (2011) Collectively rational voting rules for simple preferences. J Math Econ 47:143-149

Laslier JF, Sanver MR (eds) (2010) The basic approval voting game. In: Handbook of approval voting. Springer, New York, Chap 8, pp 153-164

Le Breton M, Weymark J (2011) Arrovian social choice theory on economic domains. In: Arrow KJ, Sen AK, Suzumura K (eds) Handbook of social choice and welfare. Amsterdam, North Holland, II, Chap 17, pp 191-299

May KO (1952) A set of independent necessary and sufficient conditions for simple majority voting. Econometrica 20:680-684

Sato N (2014) A characterization result for approval voting with a variable set of alternatives. Soc Choice Welfare. doi:10.1007/s00355-014-0811-1

Sertel MR (1988) Characterizing approval voting. J Econ Theory 45:207-211

Vorsatz M (2007) Approval voting on dichotomous preferences. Soc Choice Welfare 28:127-141

Vorsatz M (2008) Scoring rules for dichotomous preferences. Soc Choice Welfare 31:151-162

Xu Y (2010) Axiomatizations of approval voting. In: Laslier JF, Sanver MR (eds) Handbook of approval voting. Springer, New York, Chap 5, pp 91-102 